\title{
Assessment of direct-acting oral anticoagulants for the treatment of venous thromboembolism in cancer patients in Thai Tertiary Care Hospital
}

\author{
Jennis Meanwatthana ${ }^{1 *}$, Phatcharin Mitsuntisuk ${ }^{2}$ \\ ${ }_{1}$ Department of Pharmacy, Faculty of Pharmacy, Mahidol University, Bangkok, Thailand \\ 2 Pharmacy Unit, Police General Hospital, Bangkok, Thailand
}

\begin{abstract}
Venous thromboembolism (VTE) is an important cancer complication. Recent studies suggest directacting oral anticoagulants (DOACs) are possible alternatives for this population; nonetheless, there is limited evidence to support this decision in Thai cancer patients. The primary aim of this study was to measure the cumulative incidence of VTE recurrences and major bleeding among cancer patients who received DOACs that were available in Thailand. Secondary objective was to determine factors associated recurrent VTE and major bleeding. This is a retrospective cohort study conducted in tertiary care hospitals in Thailand. Data was collected from patients who had active cancer with new diagnosis of VTE and receiving approved DOACs. There were 32 cases, who received rivaroxaban, apixaban, or dabigatran, recruited to this study. We reported 4 cases $(12.5 \%)$ of recurrent VTE at 6-month. There were 6 patients (18.75\%), 2 patients $(6.25 \%)$, and 1 patient (3.13\%) with major bleeding, minor bleeding and intracranial hemorrhage, consecutively. No correlation was found between factors associated with recurrent VTE recurrence or bleeding. This study demonstrated that DOACs may be an acceptable option for preventing VTE recurrence. However, Thai population may be potentially prone to have clinically relevant bleeding. A further prospective study is warranted to draw a final conclusion in Thai cancer patients
\end{abstract}

\section{Keywords:}

Direct-acting oral anticoagulant, Cancer-associated thrombosis, VTE, Venous thromboembolism, Malignancy

\section{INTRODUCTION}

Venous thromboembolism (VTE) is a common complication in cancer patients and is considered to be a major risk of death compared to patients without cancer. Several studies have shown that the cancer associated with VTE has a three- to eight-fold mortality rate ${ }^{1-2}$. Furthermore, cancer treatment, including but not limited to certain types of anticancer and targeted therapy, is prompted by a thrombosis risk. The location of the primary tumor has been established as an independent risk factor for VTE in several studies. Specific incidence rates vary based on the clinical setting. However, pancreatic, stomach, uterine, and lung carcinomas are among the types of cancer that have been described as higher VTE incidences ${ }^{3-7}$. Besides, certain chemotherapeutic agents have been considered as an additional risk factor, such as thalidomide, or anti-vascular endothelial growth factors (VEGF) ${ }^{8}$. Moreover, there is a trivial amount of evidence of increased VTE risk in patients receiving Platinum-based chemotherapy. Additionally, treatments for cancer patients are unfortunately complicated by bleeding at higher rates than for those without cancer $^{9-10}$.

Current guidelines acquiesce to therapy options for cancer-associated thrombosis which include low molecular weight heparin (LMWH), unfractionated heparin (UFH), warfarin, and fondaparinux and direct-acting oral anticoagulants.These include the American College of Chest Physician (ACCP), the National Comprehensive

\section{*Corresponding author:}

*Jennis Meanwatthana Email: jennis.mea@mahidol.edu 
Cancer Network (NCCN), and the American Society of Clinical Oncology (ASCO $)^{11-13}$. Interestingly, there are growing evidences regarding the efficacy and safety of DOACs in this population both in clinical trials and metaanalyses ${ }^{14-23}$. DOACs are currently approved for VTE and/or stroke prevention that available in Thailand are rivaroxaban, dabigatran, apixaban, and edoxaban. Rivaroxaban, apixaban, and edoxaban are direct inhibitors of factor Xa, while dabigatran acts as a direct thrombin inhibitor. In contrast to warfarin, the DOACs do not require routine therapeutic drug monitoring, are not concerned with dietary restrictions, and are expected to have less extensive drug-drug or drug-food interactions, making these medications attractive. However, some limitations are still warranted in DOACs users specifically in chronic kidney diseases as these are mainly excreted through kidneys ${ }^{22}$. DOACs have been integrated into the treatment recommendations in all of the published guidelines in cancer associated thrombosis (CAT). In general, DOACs are recommended for patients at low risk for bleeding who do not have gastrointestinal (GI) or genitourinary $(\mathrm{GU})$ malignancies, who have adequate renal (and hepatic function, according to the NCCN guidelines), and who do not have any significant drugdrug interactions in their medical history. Patients with gastrointestinal or genital malignancies or who have had substantial drug-drug interactions should be prescribed $\mathrm{LMWH}^{12-13}$. Nonetheless, there is limited information to support this decision in Thai cancer patients.

The purpose of this study was to determine the cumulative rates of recurrent VTE and bleeding in cancer patients receiving a DOAC based on real-world evidence. We also looked into the relationship between various risk factors for VTE or bleeding, whenever possible, in order to improve awareness of anticoagulant monitoring in this patient population.

\section{MATERIALS AND METHODS}

This is a retrospective cohort study that was approved by the Institutional Review Board (IRB) committee. It was conducted in academic medical centers. The study enrolled patients with an active cancer diagnosis who were receiving Thai FDA-approved DOACs (dabigatran, apixaban, edoxaban, or rivaroxaban) for the treatment of VTE between November 2013 and November 2017. The International Statistical Classification of Diseases and Related Health Problems, 10th Revision Thai Modification (ICD-10-TM) codes were used to identify the data. The primary outcome measure was the cumulative rate of recurrent VTE in cancer patients receiving DOACs six months after starting the DOACs. Secondary outcomes included the recurrence of VTE and bleeding in patients treated with each DOAC in the study. Patients were followed for 12 months after the initial thrombotic event, and all outcomes were assessed at any point following the initial diagnosis. The demographic characteristics of the patients were presented as a percentage, mean, and standard deviation (SD). Fischer's exact test was used to compare categorical variables, and Student's unpaired t-test was used to compare continuous variables when necessary. The statistic was validated using a 0.05 two-sided alpha value. SPSS version 18 was used to conduct all data analyses (SPSS Inc., Chicago, Illinois, USA).

\section{RESULTS}

\subsection{Baseline Characteristic}

Fifty-one percent of the 32 patients included in our final report were female. These patients had an Eastern Cooperative Oncology Group (ECOG) score ranging from 0 to 2 . Rivaroxaban was the most frequently prescribed agent (16/32 cases) in our study. The majority (59.4 percent) of VTE cases occur in patients with stage III disease. The most frequently reported type of VTE was DVT in the proximal lower limb, which occurred in $50 \%$ of cases. Six patients (18.7 percent) had a prior history of anticoagulant use. Two patients were on warfarin, three were on anticoagulants for atrial fibrillation, and one had gastrointestinal bleeding. Ovarian cancer ( 9 cases; 27.3 percent) and lung cancer ( 5 cases; 15.2 percent) were the most frequently reported active cancers. At the time of the index VTE, nearly all of the patients (29/32 cases) were receiving chemotherapy. For cancer treatment, the majority of patients ( 43.8 percent and 40.6 percent, respectively) received chemotherapy using platinum complexes and/or taxane derivatives. Our study revealed comorbidities such as hypertension (22 cases; 34.9 percent), diabetes (14 cases; 22.2\%), and cardiovascular diseases (14 cases; 22.2 percent. Additionally, $25 \%$ of patients received antiplatelets or nonsteroidal anti-inflammatory drugs (NSAIDs) concurrently with DOAC initiation. The baseline characteristics of our patients are summarized in Table 1.

\subsection{Recurrent VTE and bleeding outcomes}

Cumulative VTE recurrence in DOAC-treated individuals was $12.5 \%$ at six months (4 patients). There were no new cases of VTE at the end of the year. Rethrombosis occurred after a median time of 5.4 months. Three out of four patients, according to the Khorona criteria, were categorized as "very high risk" or "high risk" for developing VTE. Among patients with recurrent VTE, two out of four cases were discovered in patients who had previously completed warfarin for provoke VTE. Following that, all patients with recurrent DVT were given LMWH. Table 2 summarizes the patient characteristics of each recurrent DVT case. There was no statistically significant difference between DOAC types 
Table 1. Patients Baseline Characteristic.

\begin{tabular}{|c|c|}
\hline Characteristics & n (\%) \\
\hline \multicolumn{2}{|l|}{ Gender } \\
\hline Female & $16(51.6)$ \\
\hline Age (Years \pm SD) & $59.6 \pm 11.3$ \\
\hline \multicolumn{2}{|l|}{ ECOG performance status (\%) } \\
\hline 0 & $25(78.1)$ \\
\hline 1 & $6(18.8)$ \\
\hline 2 & $1(3)$ \\
\hline \multicolumn{2}{|l|}{ Cancer staging } \\
\hline 2 & 7 (21.9) \\
\hline 3 & $19(59.4)$ \\
\hline 4 & $6(18.7)$ \\
\hline \multicolumn{2}{|l|}{ Type of cancers } \\
\hline Ovarian & $9(27.3)$ \\
\hline Lung & $5(15.2)$ \\
\hline Stomach & $3(9.1)$ \\
\hline Pancreas & $2(6.1)$ \\
\hline Breast & $5(15.2)$ \\
\hline \multicolumn{2}{|l|}{ Chemotherapy } \\
\hline Platinum-based compound & $14(43.8)$ \\
\hline \multicolumn{2}{|l|}{ Venous thromboembolism location } \\
\hline Pulmonary Embolism & $4(12.5)$ \\
\hline Proximal lower limb & $16(50.0)$ \\
\hline \multicolumn{2}{|l|}{ Concurrent medications } \\
\hline Antiplatelet or NSAIDs & $8(25.0)$ \\
\hline Colony stimulating factor & $9(28.0)$ \\
\hline Erythropoietin & $6(18.8)$ \\
\hline \multicolumn{2}{|l|}{ Type of Direct oral anticoagulants } \\
\hline Rivaroxaban & $16(50.0)$ \\
\hline Dabigatran & $8(25.0)$ \\
\hline Apixaban & $8(25.0)$ \\
\hline History previous anticoagulants & $6(18.8)$ \\
\hline Platelet count $\left(10^{3}\right.$ per $\left.\mathrm{uL}+\mathrm{SD}\right)$ & $179+22$ \\
\hline Serum creatinine $(\mathrm{mg} / \mathrm{dL} \pm \mathrm{SD})$ & $0.79 \pm 0.09$ \\
\hline International normalized ratio (INR \pm SD) & $0.46 \pm 0.09$ \\
\hline
\end{tabular}

ECOG-eastern cooperative oncology group

NSAIDs-non-steroidal anti-inflammatory drugs

Table 2. Patients with recurrent venous thromboembolism.

\begin{tabular}{clclclc}
\hline Case & Gender & Age (years) & Cancer type & Staging & DOACs type & Management after recurrences \\
\hline 1 & Female & 55 & Ovarian & 2 & Dabigatran & Enoxaparin \\
2 & Male & 73 & Stomach & 3 & Apixaban & Enoxaparin \\
3 & Male & 44 & Brain & 3 & Apixaban & Enoxaparin \\
4 & Female & 44 & Ovarian & 2 & Dabigatran & Enoxaparin \\
\hline
\end{tabular}

DOACs-direct-acting oral anticoagulants

in patients with recurrent DVT (Figure 1)

According to the International Society on Thrombosis and Haemostasis (ISTH) criteria, major bleeding is defined as bleeding associated with at least one of the following: transfusion of at least two units of packed red blood cells, a decrease in hemoglobin of more than two grams per deciliter, and bleeding in a critical site (intracranial, intraspinal, intraocular, pericardial, intra-articular, or intramuscular). The combined (major and non-major) bleeding rate at 6 months was $25 \%$, with major bleeding accounting for 18.8 percent. The median time to bleeding was three months. Each case of clinically relevant bleeding is described in Table 3. The gastrointestinal tract, intracranial, genitourinary, and subcutaneous tissues were the site of bleeding in our report. Bleeding occurs in patients with lung, ovary, lymphoma, and bladder cancer. There were three cases of clinically significant bleeding in which antiplatelet or non-steroidal anti-inflammatory therapy was used concurrently. Furthermore, there was no statistically significant difference in bleeding occurrence between DOAC types, as shown in Figure 1.

Additionally, we examined the association between predefined risk factors and recurrent VTE or clinically significant bleeding. The findings indicate that no correlation exists between those risks and clinical outcomes in both thrombosis and hemorrhage events. In the context of recurrent VTE, risk factors included a history of previous anticoagulant use (RR 4.33; 95 percent confidence interval [CI] 0.75-27.87; $P=0.1000)$, 
Table 3. Patients with bleeding complications.

\begin{tabular}{lclclcll}
\hline Gender & Age (years) & Cancer type & Staging & DOACs & Major bleeding & Site of bleed & $\begin{array}{l}\text { Management } \\
\text { after bleeding }\end{array}$ \\
\hline Male & 67 & Lung & 3 & Rivaroxaban & No & Bleeding gum & $\begin{array}{l}\text { DOACs } \\
\text { continued }\end{array}$ \\
\hline Male & 47 & $\begin{array}{l}\text { Non-Hodgins } \\
\text { Lymphoma }\end{array}$ & 3 & Rivaroxaban & Yes & $\begin{array}{l}\text { Gastrointestinal } \\
\text { bleeding }\end{array}$ & $\begin{array}{l}\text { Hold DOACs } \\
\text { and resumed }\end{array}$ \\
\hline Female & 53 & Ovarian & 2 & Rivaroxaban & No & Bruising & $\begin{array}{l}\text { DOACs } \\
\text { continued }\end{array}$ \\
\hline Male & 53 & Bladder & 3 & Dabigatran & Yes & Hematuria & $\begin{array}{l}\text { DOACs } \\
\text { discontinued }\end{array}$ \\
\hline Female & 65 & Lung & 4 & Rivaroxaban & Yes & $\begin{array}{l}\text { Gastrointestinal } \\
\text { bleeding }\end{array}$ & $\begin{array}{l}\text { DOACs } \\
\text { discontinued }\end{array}$ \\
\hline Female & 64 & Ovarian & 3 & Rivaroxaban & Yes & $\begin{array}{l}\text { Spontaneous } \\
\text { hematoma }\end{array}$ & $\begin{array}{l}\text { DOACs } \\
\text { discontinued }\end{array}$ \\
\hline Male & 57 & Lung & 3 & Dabigatran & Yes & $\begin{array}{l}\text { Gastrointestinal } \\
\text { bleeding }\end{array}$ & $\begin{array}{l}\text { DOACs } \\
\text { discontinued }\end{array}$ \\
\hline Male & 71 & Brain & 3 & Rivaroxaban & Yes & $\begin{array}{l}\text { Intracranial } \\
\text { Bleeding }\end{array}$ & $\begin{array}{l}\text { DOACs } \\
\text { discontinued }\end{array}$ \\
\hline
\end{tabular}

DOACs-direct-acting oral anticoagulants

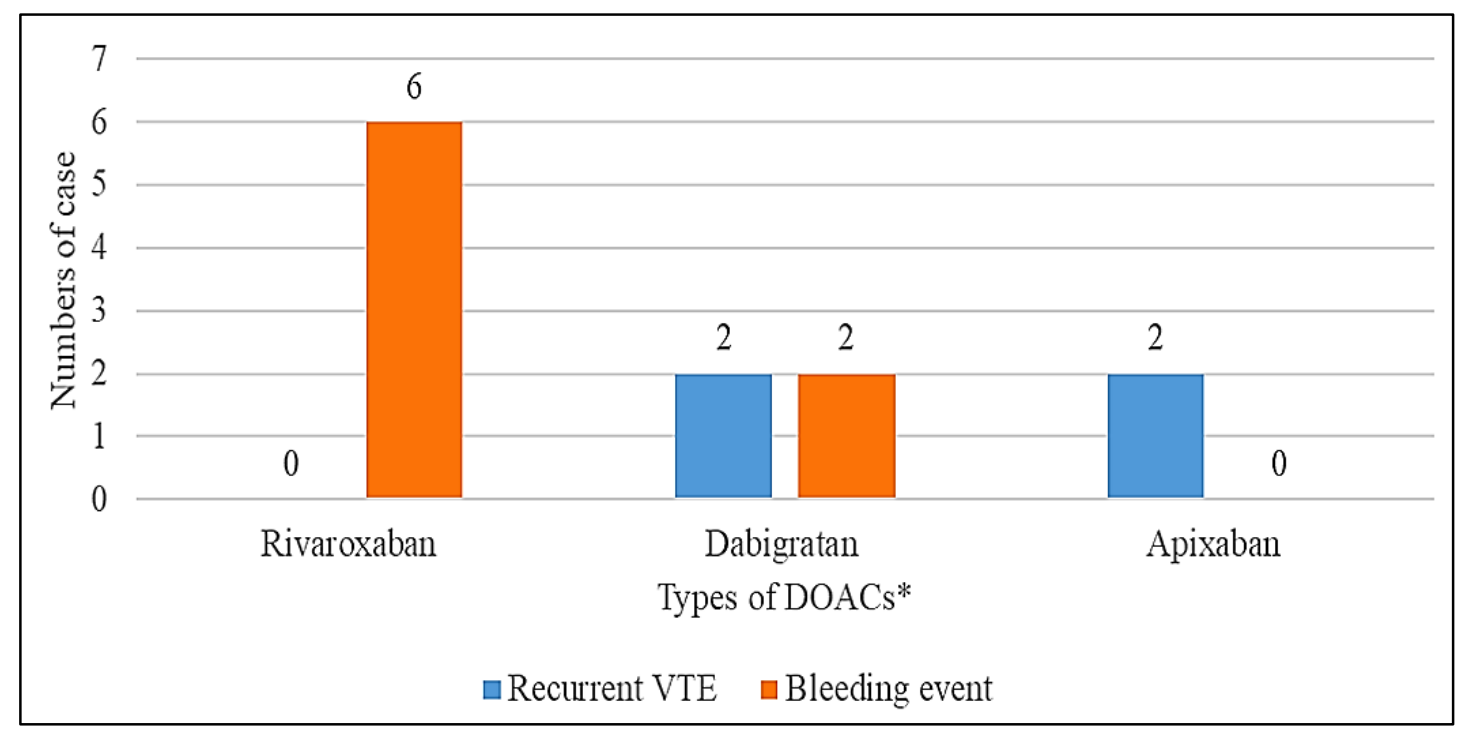

Figure 1. Number of case with reported recurrent VTE and bleeding outcomes by type of DOACs

$*=$ DOACs $=$ direct-acting oral anticoagulants

very high or high risk cancer types (RR 1.57; 95 percent CI $0.18-13.39 ; P=0.6792$ ), and use of platinum-based chemotherapy (RR 3.86; 95 percent CI 0.45-33.20; $P=0.219)$. Similarly, the RR of concurrent antiplatelet or NSAID use was 1.80 (95\% CI 0.55-5.89; $P=0.3317)$, whereas the RR of metastasis status was $0.87(95 \% \mathrm{CI}$ $0.12-6.12 ; P=0.8859)$.

\section{DISCUSSION}

Historically, subcutaneous LMWH has been the treatment of choice for secondary prophylaxis of VTE recurrence in cancer patients. There were studies including the meta-analysis compared the efficacy of LMWH to warfarin and discovered that LMWH had a significantly lower recurrence rate of VTE at six months ${ }^{22-24}$. DOACs and their approval for the treatment of VTE expand treatment options that are likely to be more tolerable, convenient for patients, and ostensibly more adherent. Although direct-acting oral anticoagulants (DOACs) are preferred for the treatment of VTE in patients without cancer, their role in cancer patients remains debatable specifically in some population ${ }^{15-17}$. Several recent studies have shown that DOACs may have an equivalent or lower incidence of recurrent VTEs compared to dalteparin, but they also have linked with a higher risk of bleeding. A previous meta-analysis, which included results from the Hokusai VTE Cancer, the SELECT-D, and the ADAM VTE trials, found that DOACs were associated with a nonstatistically significant relative risk reduction in VTE recurrence in cancer patients compared to patients treated with LMWH in cancer patients. DOACs, on the other hand, were shown to be connected with a higher risk of significant bleeding and clinical relevant non-major bleeding (CRNMB), among other things. Most current recommendations 
continue to advocate dalteparin as the first line of defense against VTEs in cancer patients, with DOAC therapy advised solely as an alternative ${ }^{16}$. More-over, in another meta-analysis, DOACs were shown to be noninferior to dalteparin in terms of reducing the risk of VTE recurrence in patients. When dalteparin was used DOACs, the risk of recurrent VTE was 1.55 times higher. More importantly, despite the fact that DOACs were linked with a higher risk of CRNMB as compared to dalteparin, the risk of major bleeding was comparable between the two treatment groups and not statistically significant difference ${ }^{18}$.

According to our retrospective findings, the majority of cases of VTE recurrence occur within the first six months following a confirmed VTE and the initiation of anticoagulation. The majority of VTE cases in our study occurred in patients with stage III cancer, which is consistent with established data demonstrating a strong association between advanced cancers and the incidence of VTE. Similarly, hemorrhagic events of clinical significance occur early in the course of treatment, regardless of the anticoagulant used. Our findings indicated slightly higher bleeding rates when compared to recent prospective data from the HOKUSAI-VTE Cancer study and the Select-D, both of which compared the use of dalteparin with edoxaban (HOKUSAI-VTE) or rivaroxaban (Select-D) in patients with cancer who had an acute VTE. As with our report, all patients enrolled in the SELECT-D trial and approximately 95\% in the Hokusai VTE Cancer trial had active cancer at the time of enrollment ${ }^{17,19}$. However, in light of recurrent VTE, the majority of our patients were diagnosed as having active cancer with a very high risk of recurrence or a high-risk type of cancer based on the Khorona classification and a history of anticoagulation, which may have contributed to the higher recurrence rate. Our overall bleeding event rate was also higher than that reported in recent studies, at $25 \%$. However, owing to the small number of cases, determining causation may be challenging. Numerous explanations may be proposed, including but not limited to the fact that individuals undergoing chemotherapy are also at risk of bleeding due to thrombocytopenia, the cancer's stage, or drugdrug interaction ${ }^{25}$. Our study's major bleeding sites were similar to those reported previously, namely the gastrointestinal tract, genitourinary system, central nervous system, and skin and soft tissue. The gastrointestinal tract was the most frequently bled area. This finding is consistent with two previous DOACs in cancer trials, which demonstrated an increased risk of gastrointestinal bleeding in patients receiving a DOAC versus conventional therapy, specifically in GI malignancy ${ }^{17-19}$. In contrast, our patients who experienced severe bleeding were from a diverse range of cancer diagnoses, which included patients with primary brain tumors as well as patients with brain metastases or hematologic malig- nancy, who were typically excluded from the various trials because they were thought to be at high risk of severe bleeding. The greater risk of bleeding seen in our research might possibly be contributed by this factor. All major bleeding events in our study necessitated the discontinuation of DOACs, and the majority were discontinued permanently regardless of the cause of the hemorrhages. However, due to the limitations of our medical record system, it was difficult to determine whether fatal bleeding was caused solely by anticoagulation.

The study's strengths include the inclusion of real-world practice in the management of VTE across the spectrum in Thai cancer patients, pre-specified and independent analysis of the study's most interesting outcomes, and the use of a uniform definition of bleeding. Nonetheless, several potential limitations of this retrospective study should be recognized. It is possible that there were cases of VTE recurrence or clinically significant VTE at other facilities during the 12-month followup period, or that there were no records in medical records. This may result in an erroneous decrease in the rate of complications. There is reason to believe that, given the retrospective nature of this study, selection bias in our cohort may have been confounded. Additionally, we reported on a small number of patients who used DOACs, which may lack the statistical power necessary to detect statistically significant and noteworthy potential risks. This is because our internal hospital consensus on cancer-related VTE is consistent with other guidelines, and our drug of choice at the moment remains LMWH, specifically enoxaparin. More importantly, the study's design incorporates numerous confounding variables that may interact with the desired outcomes. Additionally, this study was conducted in a small number of medical facilities, which may not be representative of all Thai cancer patients. However, with these concerns in mind, our current study offers essential information on the clinical out-comes of cancer associated thrombosis (CAT) which will be relevant for institutions where CAT is encountered in routine clinical practice.

\section{CONCLUSIONS}

Anticoagulation in cancer patients with VTE presents distinct concerns, including a higher risk of bleeding and recurrence of VTE than in non-cancer patients. Some clinical trial data suggest that DOACs are not inferior to LMWH in terms of preventing recurrent VTE. DOACs may be considered in Thai cancer patients who have limitations in the treatment of VTE due to LMWHs or warfarin to prevent recurrent VTE. It may be linked to an increased risk of clinically significant VTE and bleeding. DOACs should be used with caution in Thailand. To reach a final conclusion about Thai cancer patients, more prospective research is needed. 


\section{ACKNOWLEDGEMENT}

The authors thank all the participants at the hospitals for being willing to support during the recruitment phase of the study.

\section{Conflict of interests}

The authors declare that they have no conflict of interest.

\section{Funding}

This study received no external funding.

\section{Ethical Approval}

All procedures performed in studies involving human participants were in accordance with the ethical standards of the institutional research committee and with the 1964 Helsinki declaration and its later amendments or comparable ethical standards (Faculty of Dentistry/ Faculty of Pharmacy, Mahidol University, Institutional Review Board MU-DT/PY-IRB 2017/065.3010).

\section{Article info:}

Received June 18, 2021

Received in revised form December 9, 2021

Accepted December 14, 2021

\section{Informed consent}

IRB approval waiving informed consent was obtained for this retrospective study.

\section{REFERENCES}

1. Thodiyil PA, Kakkar AK. Variation in relative risk of venous thromboembolism in different cancers. Thromb Haemost. 2002; 87(6):1076-7.

2. Chew HK, Wun T, Harvey D, Zhou H, White RH. Incidence of venous thromboembolism and its effect on survival among patients with common cancers. Arch Intern Med. 2006;166(4): 458-64.

3. Monreal M, Falga C, Valdes M, Suarez C, Gabriel F, Tolosa C, et al. Fatal pulmonary embolism and fatal bleeding in cancer patients with venous thromboembolism: Findings from the RIETE registry. J Thromb Haemost. 2006;4(9):1950-6.

4. Deitcher, SR. Cancer-related deep venous thrombosis: Clinical importance, treatment challenges, and management strategies. Semin Thromb Hemost. 2003;29(3):247-58.

5. Mutirangura P, Ruengsethakit C, Wongwanit C. Epidemiologic analysis of proximal deep vein thrombosis in Thai patients: malignancy, the predominant etiologic factor. Int J Angiol. 2004; 13(2):81-3.

6. Blom JW, Doggen CJ, Osanto S, Rosendaal FR. Malignancies, prothrombotic mutations, and the risk of venous thrombosis. JAMA. 2005;293(6):715-22.

7. Khorana AA, Kuderer NM, Culakova E, Lyman GH, Francis $\mathrm{CW}$. Development and validation of a predictive model for chemotherapy-associated thrombosis. Blood. 2008;111(10):4902-7.

8. Khorana AA, Francis CW, Culakova E, Kuderer NM, Lyman GH. Frequency, risk factors, and trends for venous thromboembolism among hospitalized cancer patients. Cancer. 2007;110 (10):2339-46.

9. Streiff MB, Milentijevic D, McCrae K, Yannicelli D, Fortier J, Nelson WW, et al. Effectiveness and safety of anticoagulants for the treatment of venous thromboembolism in patients with cancer.
Am J Hematol. 2018;93(5):664-71

10. Prandoni P, Lensing AW, Piccioli A, Bernardi E, Simioni P, Girolami B, et al. Recurrent venous thromboembolism and bleeding complications during anticoagulant treatment in patients with cancer and venous thrombosis. Blood. 2002;100(10):3484-8.

11. Kearon C, Akl EA, Ornelas J, Blaivas A, Jimenez D, Bounameaux $\mathrm{H}$, et al. Antithrombotic Therapy for VTE Disease: CHEST Guideline and Expert Panel Report. Chest. 2016;149(2):315-52.

12. Streiff MB, Holmstrom B, Angelini D, Ashrani A, Elshoury A, Fanikos J, et al. Cancer-Associated Venous Thromboembolic Disease, Version 2.2021, NCCN Clinical Practice Guidelines in Oncology. J Natl Compr Canc Netw. 2021;19(10):1181-201.

13. Key NS, Khorana AA, Kuderer NM, Bohlke K, Lee AYY, Arcelus JI, et al. Venous Thromboembolism Prophylaxis and Treatment in Patients With Cancer: ASCO Clinical Practice Guideline Update. J Clin Oncol. 2020;38(5):496-520.

14. Farge D, Bounameaux H, Brenner B, Cajfinger F, Debourdeau $\mathrm{P}$, Khorana AA, et al. International clinical practice guidelines including guidance for direct oral anticoagulants in the treatment and prophylaxis of venous thromboembolism in patients with cancer. Lancet Oncol. 2016;17(10):e452-66.

15. Vedovati MC, Germini F, Agnelli G, Becattini C. Direct oral anticoagulants in patients with vte and cancer: A systematic review and meta-analysis. Chest. 2015;147(2):475-83.

16. Li A, Garcia DA, Lyman GH, Carrier M. Direct oral anticoagulant (doac) versus low-molecular-weight heparin (lmwh) for treatment of cancer associated thrombosis (cat): A systematic review and meta-analysis. Thromb Res. 2019;173:158-63.

17. Young AM, Marshall A, Thirlwall J, Chapman O, Lokare A, Hill C, et al. Comparison of an Oral Factor Xa Inhibitor With Low Molecular Weight Heparin in Patients With Cancer With Venous Thromboembolism: Results of a Randomized Trial (SELECTD). J Clin Oncol. 2018;36(20):2017-23.

18. Sabatino J, De Rosa S, Polimeni A, Sorrentino S, Indolfi C. Direct Oral Anticoagulants in Patients With Active Cancer: A Systematic Review and Meta-Analysis. JACC CardioOncol. 2020;2(3):428-40.

19. Raskob GE, van Es N, Verhamme P, Carrier M, Di Nisio M, Garcia D, et al. Edoxaban for the treatment of cancer-associated venous thromboembolism. N Engl J Med. 2018;378(7):615-24.

20. McBane Ii R, Loprinzi CL, Ashrani A, Perez-Botero J, Leon Ferre RA, Henkin S, et al. Apixaban and dalteparin in active malignancy associated venous thromboembolism. The ADAM VTE Trial. Thromb Haemost. 2017;117(10):1952-61.

21. Lutz J, Jurk K, Schinzel H. Direct oral anticoagulants in patients with chronic kidney disease: patient selection and special considerations. Int J Nephrol Renovasc Dis. 2017;10:135-43.

22. Lee AY, Levine MN, Baker RI, Bowden C, Kakkar AK, Prins $\mathrm{M}$, et al. Randomized Comparison of Low-Molecular-Weight Heparin versus Oral Anticoagulant Therapy for the Prevention of Recurrent Venous Thromboembolism in Patients with Cancer (CLOT) Investigators. Low-molecular-weight heparin versus a coumarin for the prevention of recurrent venous thromboembolism in patients with cancer. N Engl J Med. 2003;349(2):146-53.

23. Lee AYY, Kamphuisen PW, Meyer G, Bauersachs R, Janas MS, Jarner MF, et al. Tinzaparin vs warfarin for treatment of acute venous thromboembolism in patients with active cancer: a randomized clinical trial. JAMA. 2015;314(7):677-86.

24. Carrier M, Cameron C, Delluc A, Castellucci L, Khorana AA, Lee AY. Efficacy and safety of anticoagulant therapy for the treatment of acute cancer-associated thrombosis: A systematic review and meta-analysis. Thromb Res. 2014;134(6):1214-9.

25. Nakano Y, Adachi S, Imai R, Yoshida M, Shimokata S, Murohara T, et al. Mortality, Recurrent Thromboembolism and Major Bleeding in Cancer-Associated and Non-Cancer Pulmonary Embolism Patients Treated With Direct Oral Anticoagulants. Circ J. 2021. 